\title{
Editorial
}

\section{Diagnostic Approach to De-differentiated Thyroid Cancer}

\author{
El-kholy, E.
}

NCI, Cairo University. Egypt.

Thyroid carcinoma is the most prevalent endocrine malignancy and accounts for $1 \%$ of all human cancers. More than $90 \%$ of TCs are well-differentiated. ${ }^{(\mathbf{1 , 2})}$ which is defined as a carcinoma derived from the follicular epithelium and retaining basic biological characteristics of healthy thyroid tissue, including expression of the sodium iodide symporter (NIS), the key cellular feature for specific iodine uptake. It represents $70 \%$ to $90 \%$ of Thyroid Cancer and includes papillary and follicular carcinoma with all its histological variants. DTC is an uncommon disease clinically but worldwide, its incidence shows a noticeable increase ${ }^{(3)}$.

In most cases, prognosis is favorable and treatment consisting of primary surgery and ablative radioiodine administration achieves a $10-y$ survival rate of $80 \%-90 \%$ Corresponding Authors: El-kholy, E.
(4). Radioiodine is used in the management of differentiated thyroid carcinoma based on the premise that normal, as well as malignant thyroid tissues have selective uptake of radioactive iodine. It is used in various situations; diagnostic or therapeutic including ablative treatment of residual normal thyroid tissue and treatment of distant metastases ${ }^{(4-7)}$.

However when thyroid cancer cells lose their basic biological characteristics of taking up and concentrate iodine, this is called Cell De-differentiation, which is genetically based complex process that produces multiple molecular changes occurring in about $5 \%$ of cases and usually accompanied by more aggressive growth, metastatic spread, and inability to trap iodide, making the tumor resistant to radioiodine ablation and poorly responsive to conventional therapy ${ }^{(8)}$. 
Many molecular abnormalities have been detected in PTC. In adult sporadic PTC, RET/PTC rearrangements are found in 30 $40 \%$ of cases, RAS mutations in about $10 \%$, and BRAF mutations in around $40 \%$ of cases, with no overlap between these mutations (9). It is believed that the presence of the BRAFV600E mutation significantly reduces sodium-iodide symporter (NIS) expression and radioiodine uptake as well as have been associated with less differentiated and more aggressive PTC ${ }^{(\mathbf{1 0 , 1 1 )}}$.

Overexpression of tyrosine kinase (TK) receptors, and their cognate ligands, has been noted in TC cells, including receptors for epidermal growth factor and vascular endothelial growth factor (VEGF).

This fact opens a new era in therapeutic clinical trial of tyrosine kinase inhibitors for refractory thyroid cancer, which has poor prognosis and lower survivals ${ }^{(\mathbf{1 2})}$.

The reported 10-yr survival rates in dedifferentiated TC (DeTC) patients range from 49 to $68 \%$ for loco-regional recurrences and from 25 to $42 \%$ for distant metastases (12) so the known aggressive behavior, low 10 year survivals and the inability to concentrate radio-iodine leading to low diagnostic and therapeutic yield for RAI. All these negative factors making precise localization of tumor site is essential to define the therapeutic strategy. Therefore strong need for an alternative effective imaging tool that can predict and detect dedifferentiated lesions is clinically desired.

F-18 FDG PET/CT has an established role in cancer management, including thyroid cancer ${ }^{(\mathbf{1 3})}$. The fusion of the metabolic and morphologic information in PET/CT was able to increase the diagnostic accuracy, reduces pitfalls and changes therapeutic strategies in a considerable number of patients ${ }^{(14)}$.

For thyroid cancer FDG uptake and the concomitant loss of 131I uptake are known as a functional sign of dedifferentiation (15). The over expression of GLUT1 on the cell membrane of thyroid neoplasm has been shown to be closely related to more grade of dedifferentiation, which supports the clinical usefulness of FDG PET in moderate to poorly differentiated DTC with a negative radioiodine scan ${ }^{(16)}$. It was observed that the intensity of FDG uptake is correlated with progressive dedifferentiation, noting that differentiated tumors with iodine avidity have low glucose metabolism in most patients, with the converse also being true, indicating that high glucose metabolism signifies poor tumor differentiation and poor prognosis ${ }^{(16,17)}$. 
Accurate diagnostic recurrent tumor localization is very important, especially when surgical intervention is considered. In a meta-analysis of 25 studies that included 789 patients, the sensitivity of 18FDG-PET/CT was $83 \%$ (ranging from 50 to $100 \%$ ) and the specificity was $84 \%$ (ranging from 42 to $100 \%$ ) in non 131I avid TC ${ }^{(18)}$.

It was found that FDG PET is more sensitive than CT in detecting locoregional cervical nodal metastases, as malignant nodes can be early detected by PET before nodal enlargement occurs on $\mathrm{CT}^{(18)}$.

Figure 1: Shown a patient with papillary thyroid carcinoma with negative wholebody radioiodine scan after radioiodine ablation, His thyroglobulin level at time of study was $27.2 \mathrm{ng} / \mathrm{mL}$. Fused PET/CT images show recurrent thyroid papillary cancer with numerous foci of increased FDG activity consistent with recurrent disease in thyroid bed and nodal metastases in neck bilaterally.

Regarding $\mathrm{M}$ staging, FDG PET/CT is proved to be useful for detection of distant metastatic lesions, apart from pulmonary metastases that has poor sensitivity for micro-metastases less than $1 \mathrm{~cm}$ in diameter, and so if pulmonary metastases are of clinical concern, a chest CT should be performed ${ }^{(18)}$. Figure 2: Showed a patient with poorly differentiated carcinoma, underwent FDG PET/CT study after thyroidectomy, and single iodine ablation. Thyroglobulin level at time of study was 29 ng/mL. FDG PET/CT images show FDG-avid metastatic nodal, pulmonary, osseous, liver and muscular lesions. Trying to find new diagnostic approach for dedifferentiated thyroid cancer, a recent study was conducted to evaluate the role of Ga68 DOTANOC versus FDG for recurrent non iodine avid lesions and concluded that despite $68 \mathrm{Ga}$ DOTANOC PET/CT detected lesser number of lesions, yet it provides additional tool for diagnosis, and opens new vistas for treatment of dedifferentiated thyroid cancer using peptide receptor radionuclide therapy ${ }^{(\mathbf{1 9})}$.

In another study, FDG PET/MRI was inferior to low-dose FDG PET/CT for the assessment of pulmonary status in patients with dedifferentiated thyroid cancer. However, for the assessment of cervical regional lymph node, FDG PET/MRI was equal to contrast-enhanced neck FDG PET/CT ${ }^{(20)}$. 
Therefore, FDG PET/MRI combined with a low-dose CT scan of the thorax may provide an imaging solution when highquality imaging is needed and high-energy CT is undesirable or the use of a contrast agent is contraindicated. Finally, integrated PET/CT is able to improve diagnostic accuracy in a therapeutically relevant way in patients with deDTC.
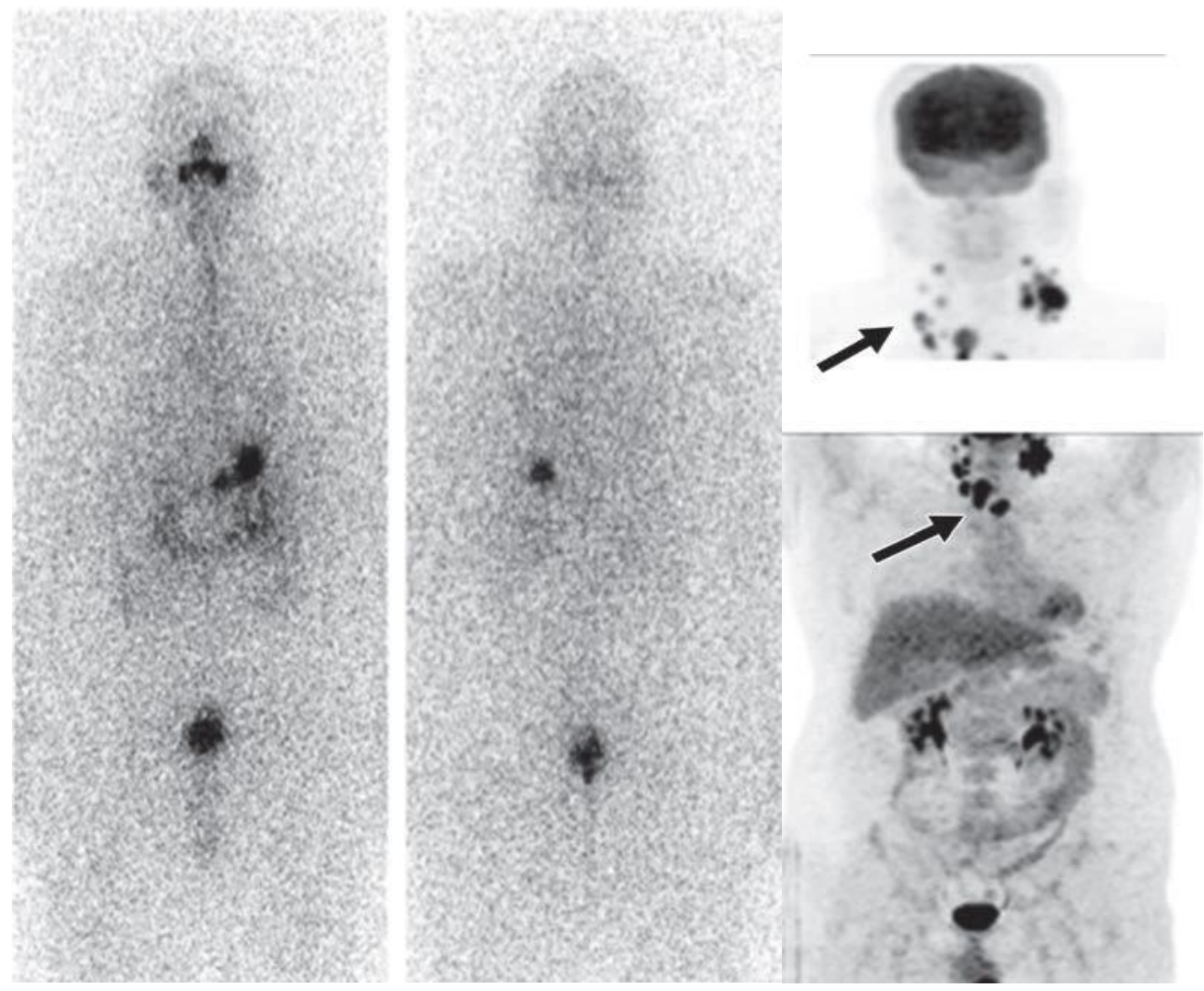

Figure (1): 37-year-old man with papillary thyroid carcinoma with negative whole-body radioiodine scan after radioiodine ablation, His thyroglobulin level at time of study was 27.2 ng/mL. Fused PET/CT images show recurrent thyroid papillary cancer with numerous foci of increased FDG activity consistent with recurrent disease in thyroid bed and nodal metastases in neck bilaterally. 


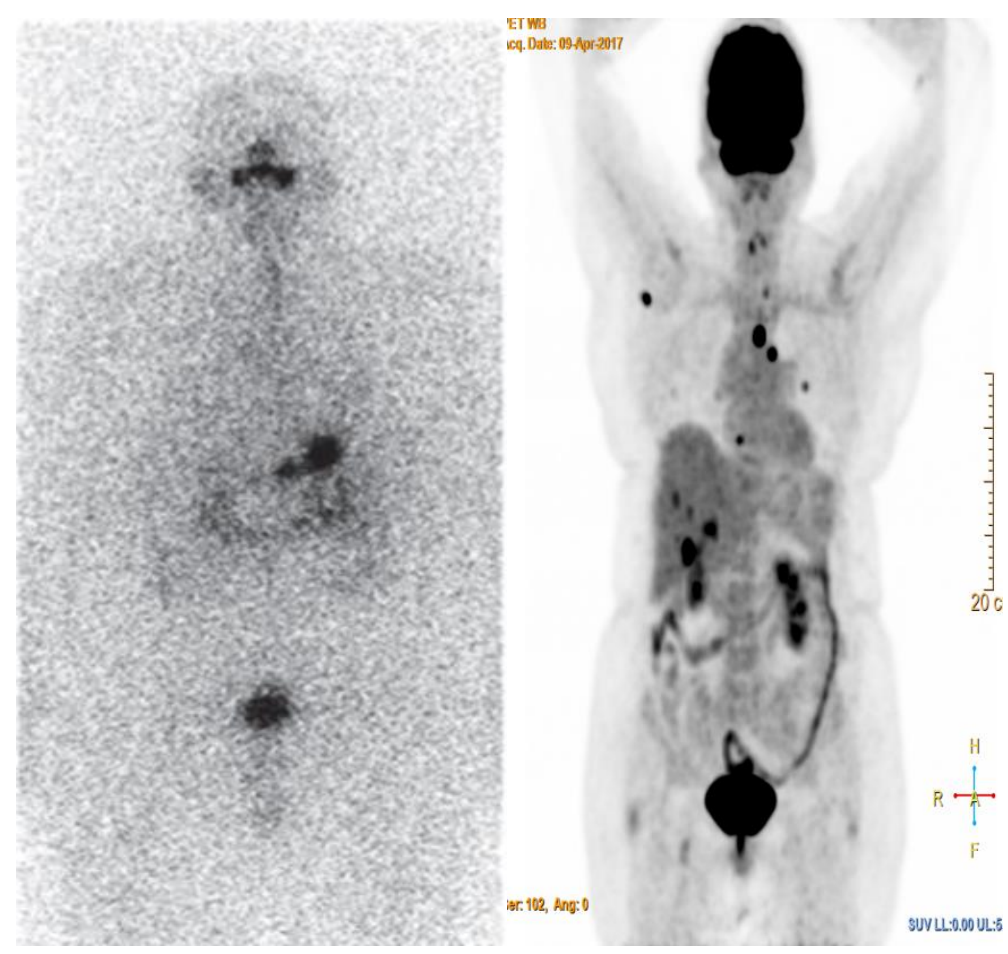

Figure (2): 70 -year-old Female patient with poorly differentiated carcinoma underwent FDG PET/CT study after thyroidectomy, and single iodine ablation. Thyroglobulin level at time of study was $29 \mathrm{ng} / \mathrm{mL}$. FDG PET/CT images show FDG-avid metastatic nodal, pulmonary, osseous, liver and muscular lesions.

\section{REFRENCES:}

1) Mazzaferri, E. L. and Massoll, N. "Management of papillary and follicular (differentiated) thyroid cancer: new paradigms using recombinant human thyrotropin," Endocrine-Related Cancer, vol. 9, no. 4, pp. 227-247; 2002.

2) Schlumberger, M. J. "Papillary and follicular thyroid carcinoma, The New England Journal of Medicine, vol. 338, no. 5, pp. 297-306; 1998.
3) Hodgson NC, Button J, Solorzano $\boldsymbol{C} \boldsymbol{C}$. Thyroid cancer: is the incidence still increasing? Ann. Surg. Oncol. 11:1093-7; 2004.

\section{4) Rodrigues $F$, Limbert E, Marques} AP, et al. Grupo de Estudo da Tiróide.et al. [Treatment and follow up protocol in differentiated thyroid carcinomas of follicular origin]. Acta. Med. Port.18:2$16 ; 2005$. 
5) Pacini, F. Schlumberger, M. Dralle

$\boldsymbol{H}$, et al. European consensus for the management of patients with differentiated thyroid carcinoma of the follicular epithelium. Eur. J. Endocrinol.154:787803; 2006.

\section{6) Cooper, DS. Doherty GM, Haugen}

H.et al. Management guidelines for patients with thyroid nodules and differentiated thyroid cancer. Thyroid. 16:109-42; 2006.

7) Dietlein, M. Eschner, W. Grünwald

$\boldsymbol{F}$, et al. Procedure guidelines for radioiodine therapy of differentiated thyroid cancer (version 3).

Nuklearmedizin. 46:213-9; 2007.

8) Haugen, B. $\boldsymbol{R}$ "Management of the patient with progressive radioiodine nonresponsive disease," Seminars in Surgical Oncology, vol. 16, no. 1, pp. 34-41; 1999.

\section{9) Baudin, E and Schlumberger $M$.}

New therapeutic approaches for metastatic thyroid carcinoma. Lancet. Oncol. 8:148156; 2007.
10) Sarlis, NJ and Benvenga $S$. Molecular signaling in thyroid cancer. In: Farid NR, ed. Molecular basis of thyroid cancer. Boston: Kluwer; 236-263; 2004.

11) Krause, DS and VanEtten, RA. Tyrosine kinases as targets for cancer therapy. N. Engl. J. Med. 353:172-187; 2005.

12) Baudin, E and Schlumberger, $M$. New therapeutic approaches for metastatic thyroid carcinoma. Lancet. Oncol. 8:148156; 2007.

13) Iagaru, A. Kalinyak, JE and McDougall IR. F-18 FDG PET/CT in the management of thyroid cancer. Clin. Nucl. Med. Sep; 32:690-5; 2007.

14) Lind, $P$ and Kohlfürst, $S$. Respective roles of thyroglobulin, radioiodine imaging, and positron emission tomography in the assessment of thyroid cancer. Semin. Nucl. Med. Jul; 36:194205; 2006. 
15) Lietzenmaye,r R. Muller-Ber,g M and Thelen MH, et al. Follow-up after radioiodine treatment of differentiated thyroid cancer [in German; abstract]. Nuklearmedizin.37:A23; 1998.

16) Feine, U. Lietzenmayer, $R$ and Hanke, JP, et al. Fluorine-18-FDG and iodine-131-iodide uptake in thyroid cancer. J. Nucl. Med. 37(9):1468-72; 1996.

17) Jadvar, $H$ and Parker, J.A. Oncology-Head, neck, and thyroid. In: Clinical PET and PET/CT. ISBN 1-85233838-5. Springer. pp 107-109; 2006.
18) Alavi, A and Eugene, C. LinVincent. PET \& PET CT A clinical guide. 2nd ed. ISBN: 978-1-60406-135-6. Thieme Medical Publisher, Inc. pp 136-141; 2009.

19) Kundu, P. Lata, S and Jeph, S et al. Role of $68 \mathrm{Ga}-\mathrm{DOTANOC} \mathrm{PET/CT}$ in dedifferentiated thyroid cancer and comparison with 18FDG PET/CT. J Nucl Med.; 52 (Supplement 1):182; 2011.

20) Vrachimis, A. Burg, $M$ and Wenning, $\quad C$. [18F] FDG PET/CT outperforms $[18 \mathrm{~F}]$ FDG PET/MRI in differentiated thyroid cancer. EJNMMI. 43, 2: pp 212-220; 2015. 